Article

\title{
A Method for Identification of Driving Patterns in Hybrid Electric Vehicles Based on a LVQ Neural Network
}

\author{
Hongwen He *, Chao Sun * and Xiaowei Zhang
}

National Engineering Laboratory for Electric Vehicles, Beijing Institute of Technology, Beijing 100081, China; E-Mail: fot8150@126.com

* Authors to whom correspondence should be addressed; E-Mails: hwhebit@bit.edu.cn (H.H.); sunchao305373850@gmail.com (C.S.); Tel./Fax: +86-10-6891-4842 (H.H.)(C.S.).

Received: 9 July 2012; in revised form: 17 August 2012 / Accepted: 24 August 2012 /

Published: 5 September 2012

\begin{abstract}
Driving patterns exert an important influence on the fuel economy of vehicles, especially hybrid electric vehicles. This paper aims to build a method to identify driving patterns with enough accuracy and less sampling time compared than other driving pattern recognition algorithms. Firstly a driving pattern identifier based on a Learning Vector Quantization neural network is established to analyze six selected representative standard driving cycles. Micro-trip extraction and Principal Component Analysis methods are applied to ensure the magnitude and diversity of the training samples. Then via Matlab/Simulink, sample training simulation is conducted to determine the minimum neuron number of the Learning Vector Quantization neural network and, as a result, to help simplify the identifier model structure and reduce the data convergence time. Simulation results have proved the feasibility of this method, which decreases the sampling window length from about 250-300 s to $120 \mathrm{~s}$ with an acceptable accuracy. The driving pattern identifier is further used in an optimized co-simulation together with a parallel hybrid vehicle model and improves the fuel economy by about $8 \%$.
\end{abstract}

Keywords: hybrid electric vehicles; LVQ; neural network; driving pattern recognition; simulation; fuel economy 


\section{Introduction}

The use of hybrid electric vehicles (HEVs) is one of the most effective ways to improve fuel economy and reduce vehicle emissions. Since the powertrain control strategy plays a very important role in improving the performance of HEVs, the study of new control theories that can be applied to the power trains of HEVs is very significant $[1,2]$. Many control strategies, such as fuzzy control, logic threshold control, dynamic programming and artificial neural network control, have been developed and successfully applied in HEVs [2-10]. Comparative studies have shown that the flexibility of the control strategies is very important for the improvement of the control effects [2,3]. This paper attempts to achieve an identification of vehicle driving patterns to improve the control flexibility, and eventually improve the fuel economy of HEVs.

A kind of multi-mode driving control strategy has been accomplished in [4,5] through applying the concept of driving pattern recognition (DPR). DPR can provide integrated driving information for the vehicle main controller to make decisions and the control strategy can be more adaptive to a variety of driving conditions. There are two main methods to identify vehicle driving patterns at present. One is to predict future conditions using the traffic environment information provided by $3 \mathrm{~S}$ (GPS, GIS, ITS) [6-8]. The other is to predict future conditions through analyzing the driving information gathered by vehicle-carrying sensors. As the second type of driving pattern identification method relies only on the theoretical study of the control algorithms, which can be applied more easily, this paper focuses only on method two [9]. Lin et al. [5] and Won et al. [10] have both realized a kind of DPR based on the analysis of feature parameters extracted from the velocity data. The control effects are impressive.

Langari and Won proposed an intelligent energy management agent (IEMA) for parallel hybrid vehicles, which contained a Learning Vector Quantization (LVQ) network roadway type identifier [11]. During their research, 47 parameters were selected for LVQ classification and most of the drive cycle segments could be correctly classified [12].

Lei et al. analyzed the impacts on identification results caused by the dissimilarity measures used in DPR, including the Euclidean distance, Chebyshev distance, cosine distance, correlation distance and Mahalanobis distance [13]. Research results show that realizing DPR through calculating the Euclidean distance is more adaptable [13,14]. In fact, the LVQ neural network recognition algorithms are exactly based on the calculation of Euclidean distances. This paper is based on the researches of LVQ recognition above, and new mathematical methods are been used to increase the identification performance of LVQ network.

The selection of representative features has a great impact on the effect of DPR. Sixty two driving parameters that might have influences on fuel economy and emissions of HEVs have been studied in [15], concluding that nine of them had a great effect. In subsequent studies, the number of representative features, though selected differently, has tended to decrease. For example, [4] used about 24 features for DPR in 2002, but [16] and [11] decreased them to 17 and 15 in 2009 and 2012 respectively.

In this paper, micro-trip extraction and Principal Component Analysis (PCA) methods are applied to increase the LVQ training sample quantity and reduce the input vector dimension. The number of representative features for principal component analysis is reduced to seven. As a result, the LVQ 
neural network pattern recognition model is optimized and the recognition accuracy is improved with less computing space and sampling time.

Figure 1 demonstrates the HEV control logic based on LVQ driving pattern recognition suggested in this paper. The control parameters of several specific driving cycles are optimized to develop a logic threshold library. When a certain driving cycle is identified, the corresponding control parameters will be selected from the logic threshold library and updated into the ECU in real time.

Figure 1. Control logic based on DPR.

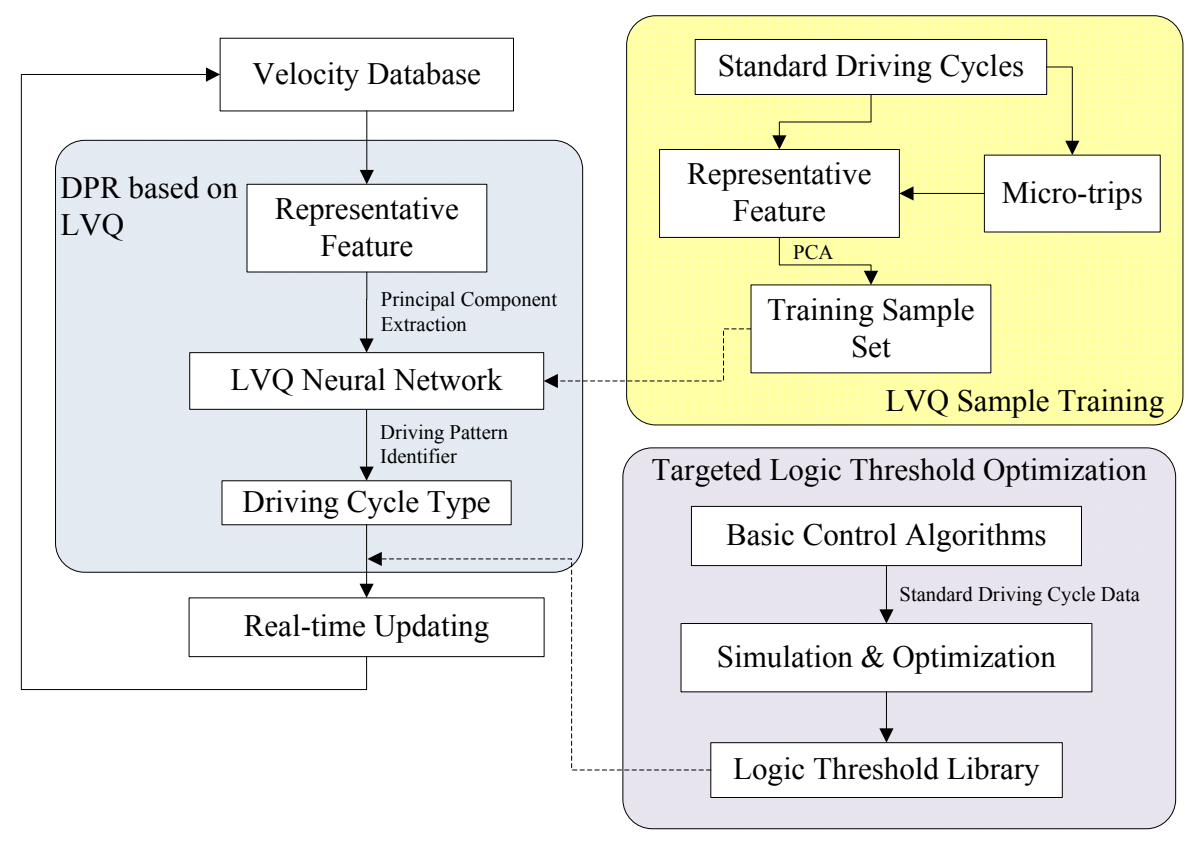

\section{Driving Cycle Analysis and Training Sample Collection}

The target of DPR is to analyze the velocity information and classify practical driving patterns as similar standard driving cycles. Recognized driving cycles should be included in the representative driving cycle group. Six representative standard driving cycles, shown in Table 1 and Figure 2a-f, were selected in this paper to cover most of the different street types, driving behaviors and weather conditions.

Table 1. Representative standard driving cycles.

\begin{tabular}{ccc}
\hline Driving Cycle Type & Name & Driving Cycle Number \\
\hline \multirow{2}{*}{ Urban Road } & MANHATTAN & Driving Cycle 1 \\
& NYCC & Driving Cycle 2 \\
\hline \multirow{2}{*}{ Suburb Road } & WVUSUB & Driving Cycle 3 \\
& CSHVR & Driving Cycle 4 \\
\hline \multirow{2}{*}{ Highway Road } & HWFET & Driving Cycle 5 \\
& HS06_HWY & Driving Cycle 6 \\
\hline
\end{tabular}


Figure 2. Velocity-time profiles of the six representative cycles; (a) MANHATTAN;

(b) NYCC; (c) WVUSUB; (d) CSHVR; (e) HWFET; (f) US06_HWY.

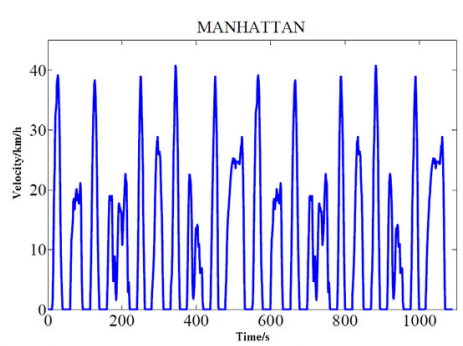

(a)

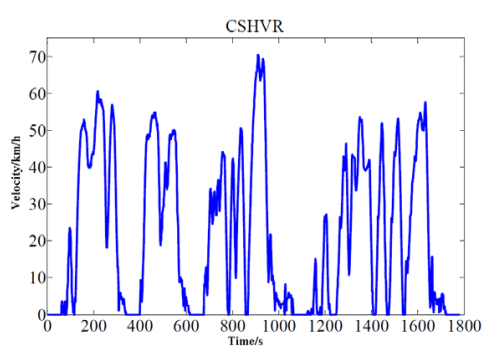

(d)

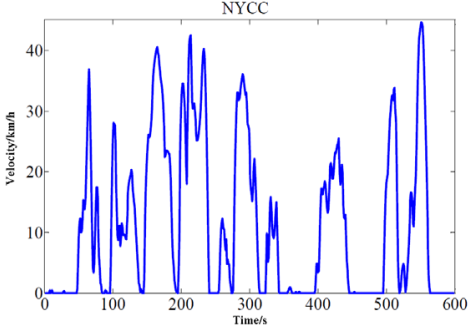

(b)

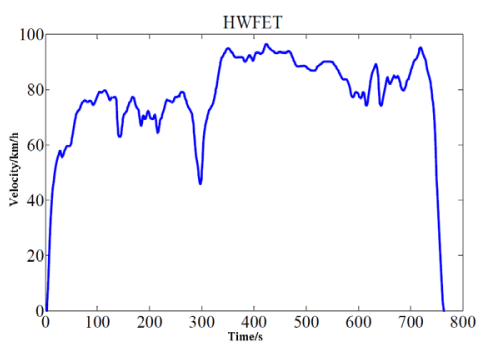

(e)

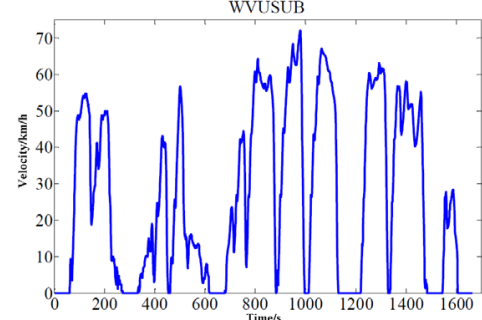

(c)

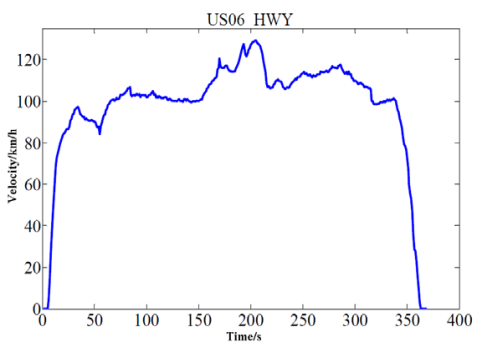

(f)

\subsection{Representative Feature Analysis for DPR}

Seven representative features are selected for PCA, in which four principal components are eventually retained. The principal components are imported as inputs of the driving pattern identifier which is based on LVQ neural network.

\subsubsection{Representative Features}

Average cycle speed:

$$
V_{a v g}=\frac{\int v d t}{t}
$$

Maximum cycle speed:

$$
V_{\max }=\max \left(v_{i}, i=1,2, \cdots, k\right)
$$

Maximum cycle acceleration:

$$
a_{a \max }=\max \left(a_{a i}, 1,2, \cdots, k\right)
$$

Maximum cycle deceleration:

$$
a_{d \max }=\max \left(a_{d i}, i-1,2, \cdots, k\right)
$$

Average acceleration:

$$
a_{\text {aavg }}=\frac{\int\left|a_{a}(t)\right| \cdot d t}{T_{\text {trip }}}
$$


Average deceleration:

$$
a_{\text {davg }}=\frac{\int\left|a_{d}(t)\right| \cdot d t}{T_{\text {trip }}}
$$

Percentage of idle time:

$$
i d_{\text {per }}=\frac{T_{\text {idel }}}{T_{\text {trip }}} \times 100 \%
$$

where: $k$-sampling point number; $T_{\text {trip }}$ — cycle total time. Calculation results of the representative features are shown in Table 2.

Table 2. Representative features of six standard driving cycles.

\begin{tabular}{lcccccc}
\hline & MANHATTAN & NYCC & WVUSUB & CSHVR & HWFET & US06_HWY \\
\hline$V_{\text {avg }}$ & 10.98 & 11.41 & 25.87 & 21.86 & 77.58 & 97.91 \\
$V_{\max }$ & 40.72 & 44.58 & 72.10 & 70.49 & 96.40 & 129.23 \\
$a_{a \max }$ & 2.06 & 2.68 & 1.29 & 1.16 & 1.43 & 3.08 \\
$a_{d_{\max }}$ & -2.5 & -2.64 & -2.16 & -1.79 & -1.48 & -3.08 \\
$a_{\text {aavg }}$ & 0.54 & 0.62 & 0.33 & 0.39 & 0.19 & 0.34 \\
$a_{\text {davg }}$ & -0.67 & -0.61 & -0.42 & -0.46 & -0.22 & -0.41 \\
$i d_{\text {per }}$ & $36.18 \%$ & $35.12 \%$ & $25.23 \%$ & $21.63 \%$ & $0.78 \%$ & $3.26 \%$ \\
\hline
\end{tabular}

\subsubsection{Principal Component Analysis}

Suppose that the sample data matrix is $X$ and the sample correlation coefficient matrix is $R$. The principal component matrix would be $Z=R X$. Considering $i=1,2, \cdots, 7$ and $j=1,2, \cdots, 6$.

According to Table 2, the sample data matrix $X$ is:

$$
X_{i j}=\left[\begin{array}{cccc}
x_{11} & x_{12} & \cdots & x_{16} \\
x_{21} & x_{22} & \cdots & x_{26} \\
\vdots & \vdots & \ddots & \vdots \\
x_{71} & x_{72} & \cdots & x_{76}
\end{array}\right]
$$

This can be standardized as:

$$
X_{i j}^{*}=\frac{x_{i j}-\bar{x}_{j}}{\sqrt{\operatorname{var}\left(x_{j}\right)}}
$$

where $\bar{x}_{j}=\frac{1}{7} \sum_{i=1}^{7} x_{i j}$ and $\operatorname{var}\left(x_{j}\right)=\frac{1}{7-1} \sum_{i=1}^{7}\left(x_{i j}-\bar{x}_{j}\right)^{2}$.

The sample correlation coefficient matrix is:

$$
R=\left[\begin{array}{cccc}
r_{11} & r_{12} & \cdots & r_{16} \\
r_{21} & r_{22} & \cdots & r_{26} \\
\vdots & \vdots & \ddots & \vdots \\
r_{61} & r_{62} & \cdots & r_{66}
\end{array}\right]
$$


Suppose the standardized original data matrix is still represented by $X$, the standardized correlation coefficient of the data can be calculated as:

$$
r_{i j}=\frac{\sum_{k=1}^{6}\left(x_{k i}-\overline{x_{i}}\right)\left(x_{k j}-\overline{x_{j}}\right)}{\sqrt{\sum_{k=1}^{6}\left(x_{k i}-\overline{x_{i}}\right)^{2} \sum_{k=1}^{6}\left(x_{k j}-\overline{x_{j}}\right)^{2}}}
$$

where $i, j=1,2, \cdots, 6$.

The Jacobi iteration method is applied to compute the eigenvalue $\left(\lambda_{1}, \lambda_{2}, \cdots, \lambda_{6}\right)$ of the correlation coefficient matrix $R$ and the corresponding eigenvectors $a_{i}=\left(a_{i 1}, a_{i 2}, \cdots, a_{i 6}\right)$.

The contribution rate of the principal component is:

$$
R_{\text {ATIO }} \text { CONTRIBUTION }=\frac{\lambda_{i}}{\sum_{i=1}^{6} \lambda_{i}}
$$

Principal component matrix $Z$ can be calculated as:

$$
\left\{\begin{array}{c}
Z_{1}=a_{11} x_{1}+a_{12} x_{2}+\cdots+a_{16} x_{6} \\
Z_{2}=a_{21} x_{1}+a_{22} x_{2}+\cdots+a_{26} x_{6} \\
\cdots \cdots \\
Z_{p}=a_{p 1} x_{1}+a_{p 2} x_{2}+\cdots+a_{p 6} x_{6}
\end{array}\right.
$$

Calculation results of the principal component matrix $Z$ are shown in Table 3. Six principal components can be obtained by PCA $\left(Z_{1}-Z_{6}\right)$. The entire principal components are rearranged according to the descending of the CONTRIBUTION RATIO, as shown in Figure 3. The maximum four principal components (accumulative ratio $=98.92 \%$ ) are finally selected as inputs of the identifier.

Figure 3. CONTRIBUTION RATIO distribution of the principal components.

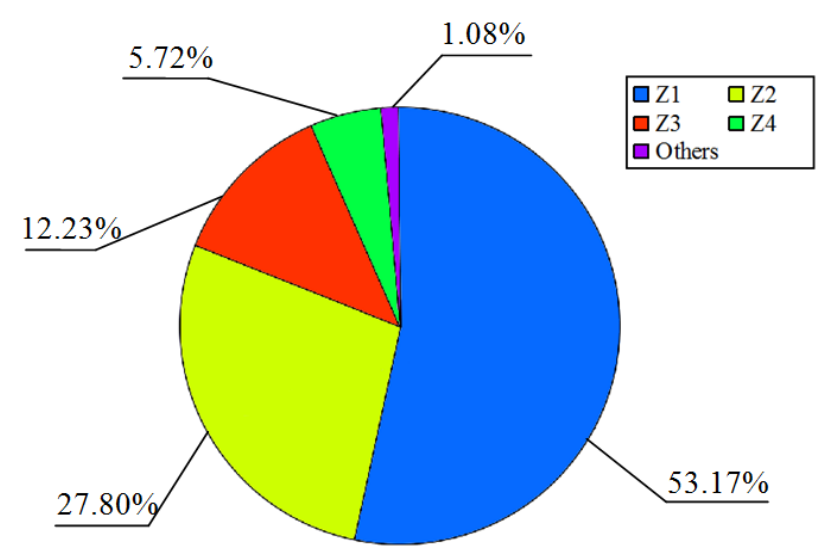


Table 3. Calculation results of the principal component matrix $Z$.

\begin{tabular}{ccccccc}
\hline$a_{i j}$ & $\mathbf{x}_{\mathbf{1}}$ & $\mathbf{x}_{\mathbf{2}}$ & $\mathbf{x}_{\mathbf{3}}$ & $\mathbf{x}_{\mathbf{4}}$ & $\mathbf{x}_{\mathbf{5}}$ & $\mathbf{x}_{\mathbf{6}}$ \\
\hline$Z_{1}$ & -0.4161 & 0.3039 & 0.1756 & -0.2419 & -0.4373 & 0.6574 \\
$Z_{2}$ & -0.4181 & 0.2808 & -0.3934 & -0.1847 & 0.6006 & 0.1368 \\
$Z_{3}$ & 0.0801 & 0.6356 & 0.5199 & 0.2099 & -0.0885 & -0.2639 \\
$Z_{4}$ & -0.1655 & -0.5997 & 0.5562 & -0.2915 & 0.1059 & 0.0873 \\
$Z_{5}$ & 0.4481 & 0.1735 & 0.3656 & -0.1302 & 0.5942 & 0.3736 \\
$Z_{6}$ & -0.4516 & -0.1502 & 0.2040 & 0.7894 & 0.2349 & 0.1333 \\
\hline
\end{tabular}

\subsection{Micro-Trip Extraction and Sample Collection}

A sufficient number of samples can ensure the training effect and ultimately affect the DPR results. Micro-trip extraction can increase the number of training samples greatly. Micro-trips refer to a continuously driving period from an idle segment to the next idle segment [17-19].

Considering driving cycles 1-4, the two urban road cycles and two suburb road cycles, each of them contains at least seven micro-trips, which can be collected for statistical analysis. The velocity information collection window length is determined according to the concentrated length of the operating time of most micro-trips. Researches show that the operating time of $80 \%$ of the micro-trips maintains within 120 seconds [20], as shown in Figure 4.

Figure 4. Statistics of micro-trips of driving cycle 1-4.

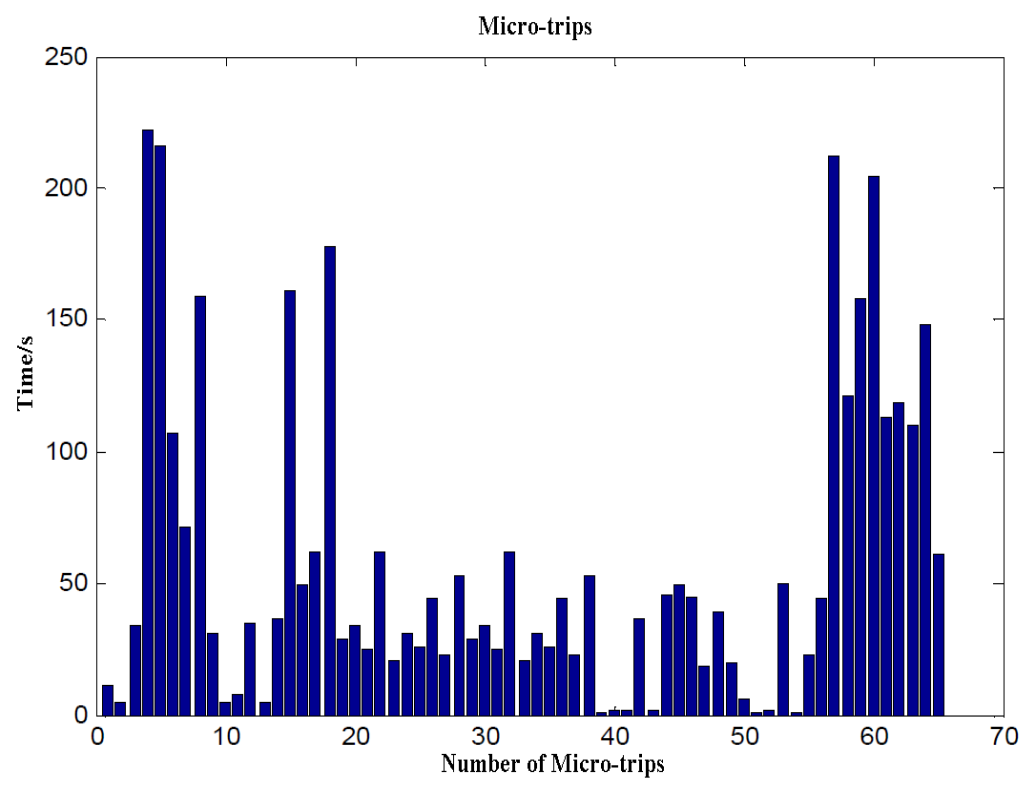

However, the two highway driving cycles, driving cycles 5-6, cannot be segmented in terms of idling time, because there are few, or even no idling periods. This paper proposes a method to divide highway driving cycles into seven micro-trips. As illustrated in Figure 5, driving cycle 5 is quartered to obtain four micro-trips (1-4) first, and the midpoints of adjacent micro-trips can shape three other micro-trips (5-7). The same method is used to partition driving cycle 6. Representative features of each micro-trip will be imported as training sample data. Thus, the entire number of the training samples increases from 6 to 85, which can provide an essential precondition for high-accuracy recognition. 
Figure 5. Micro-trip partition of driving cycle 5.

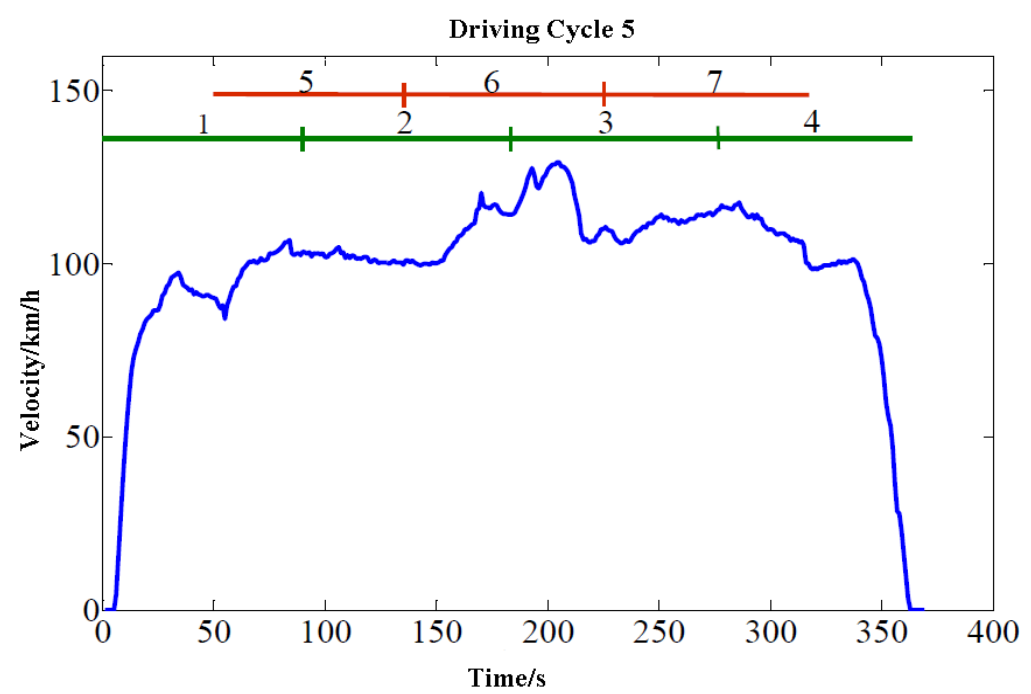

\section{Driving Pattern Recognition based on LVQ Neural Network}

\subsection{LVQ Neural Network}

An LVQ network can adjust the weights of the competition layer through self-organization, with a large number of training samples imported, to reflect the distribution of the sample data patterns in the output layer [21]. LVQ pattern recognition algorithm evolved from the Kohonen competitive algorithm and has been applied widely in the field of pattern recognition [22]. The architecture of the LVQ network is as illustrated in Figure 6 [12,23].

Figure 6. Architecture of LVQ neural network.

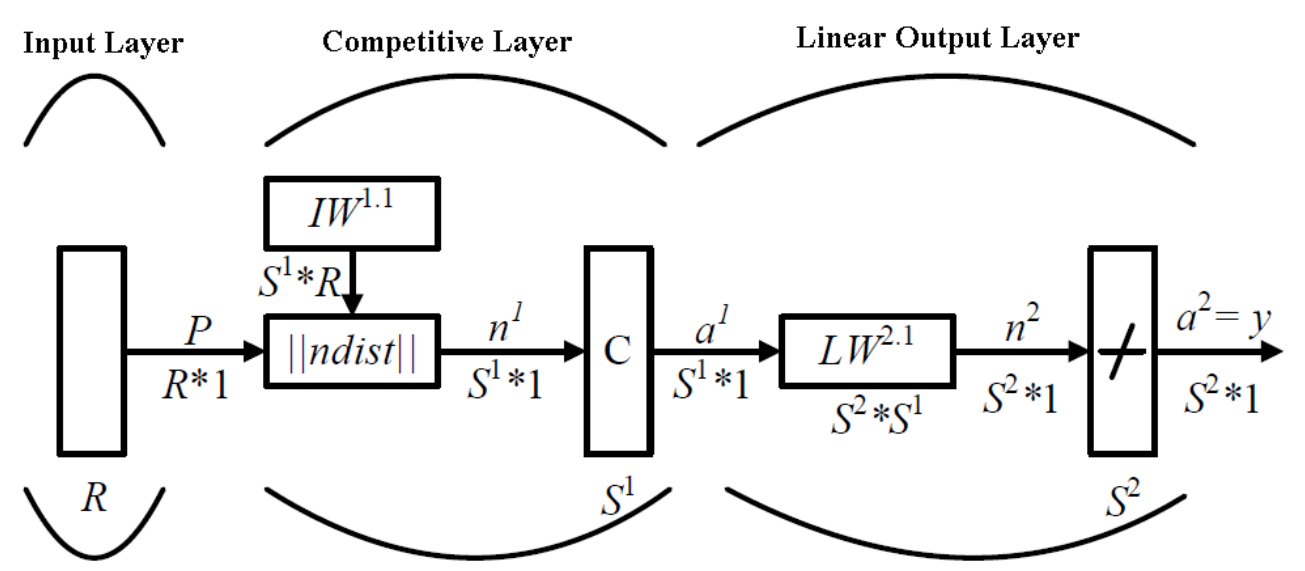

where: $p-R$-dimensional input; $S^{1}$-number of the competition layer neurons; $I W^{1.1}$-connection weight coefficient matrix between input and competition layer; $n^{1}$-input of competition layer neuron; $a^{1}$-output of competition layer neuron; $L W^{2.1}$ — connection weight coefficient matrix between competition layer and linear output layer; $n^{2}$-input of linear output layer neuron; $a^{2}$ - output of linear output layer neuron; $n^{1}=-\left\|I W^{1.1}-p\right\| ; n^{2}=L W^{2.1} * a^{1} ; a^{1}=\operatorname{compet}\left(n^{1}\right) ; a^{2}=\operatorname{purelin}\left(n^{2}\right)$. 


\subsection{Driving Pattern Identifier Model}

\subsubsection{Model Structure}

A driving pattern identifier model is established via Matlab/Simulink as shown in Figure 7. The LVQ neural network can classify input vectors into one of the target patterns and output recognition results directly. The competition layer is used to identify the subclasses of input vectors and the subclasses are all connected to the final target patterns or classes through linear output layer. By calculating the Euclidean distances of input vectors and the competition layer neurons, neurons in the competition layer can classify input vectors into subclasses rapidly. The closest competition neurons are marked as " 1 " and others as "0".

Figure 7. Identifier model based on LVQ neural network.

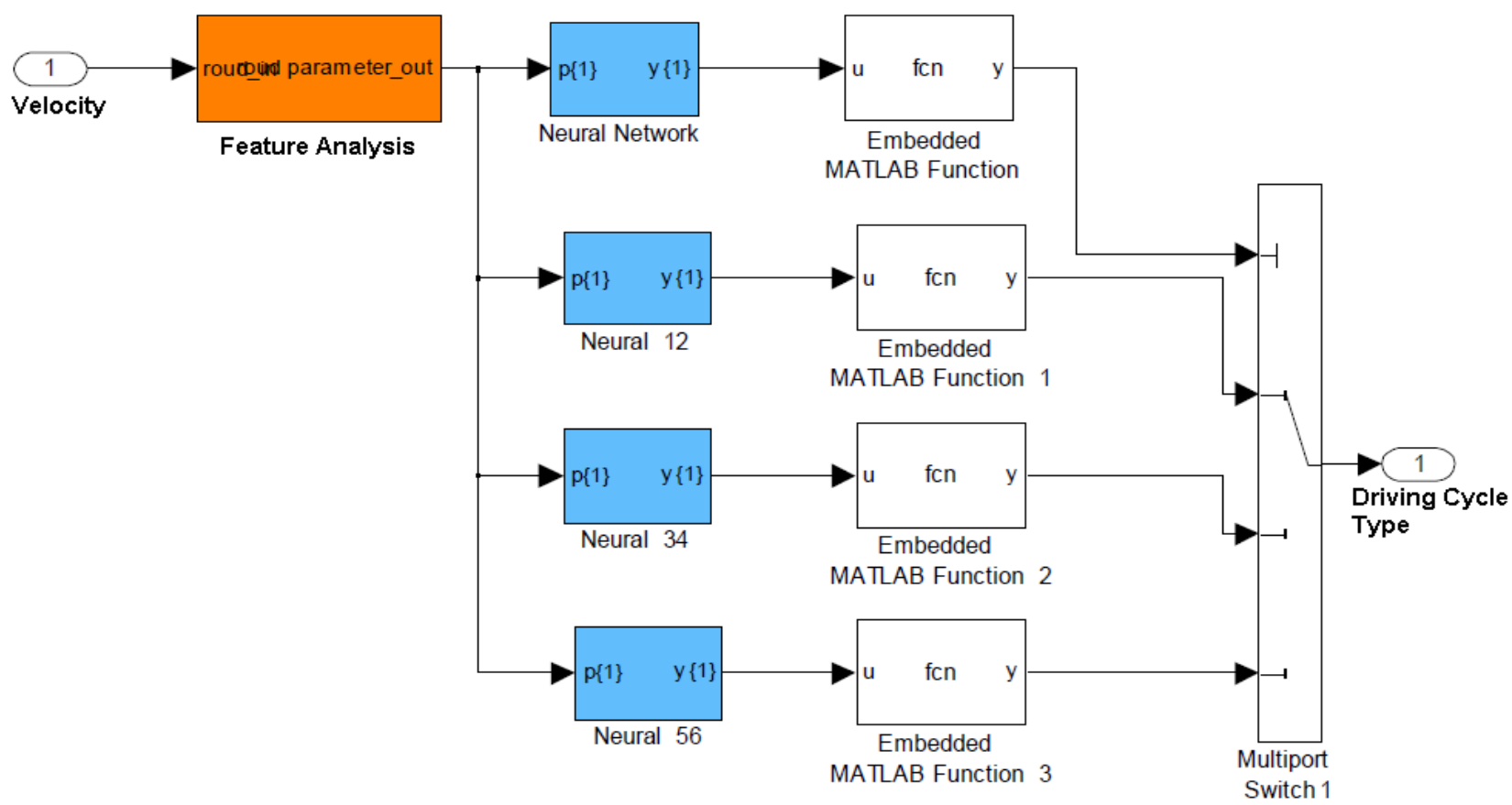

Figure 8 shows the internal structure of the driving pattern identifier. The upper section is the computing process model from input layer to competition layer and the second section is the computing process model from competition layer to linear output layer. There are four importing nodes corresponding to the four principal components calculated by the seven characteristic parameters of the velocity data. To be more specific, the neurons of the competition layer are used to classify input vectors through competitive learning and the neurons of linear output layer are used to transfer the classification information, which will be delivered from competition layer, into the defined expectation categories. Though within a certain range, the more neurons there are, the more accurate the pattern recognition will be. The recognition effect could not be improved obviously when the neuron number is above five and the training time will become much longer according to the simulation conducted for neuron number optimization. The number of the neurons in the competition layer is set as four in this paper via a three-time sample training simulation and the number of the neurons in linear output layer is set as six because six kinds of standard cycles are expected to be identified by LVQ neural network. 
Figure 8. Internal structure of the driving pattern identifier.

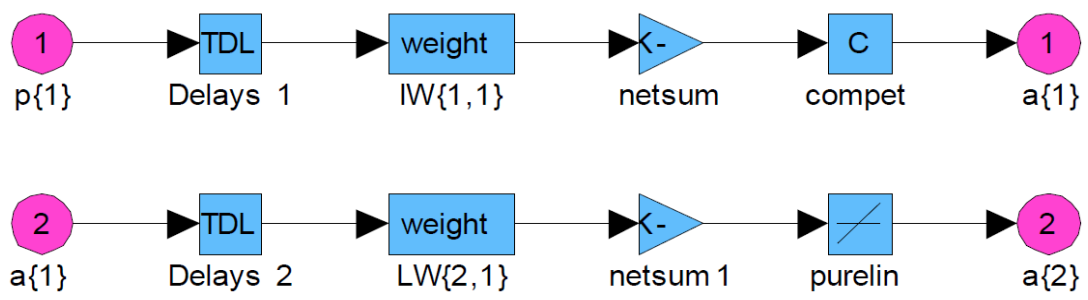

\subsubsection{Sample Training}

Principal components computed from the characteristic parameters of six standard driving cycles and 79 micro-trips are imported as training sample data. The training process is under supervision and the competition layer weights are adjusted continually according to the learning results of the LVQ neural network, as shown in Figure 9.

Figure 9. Sample training process.

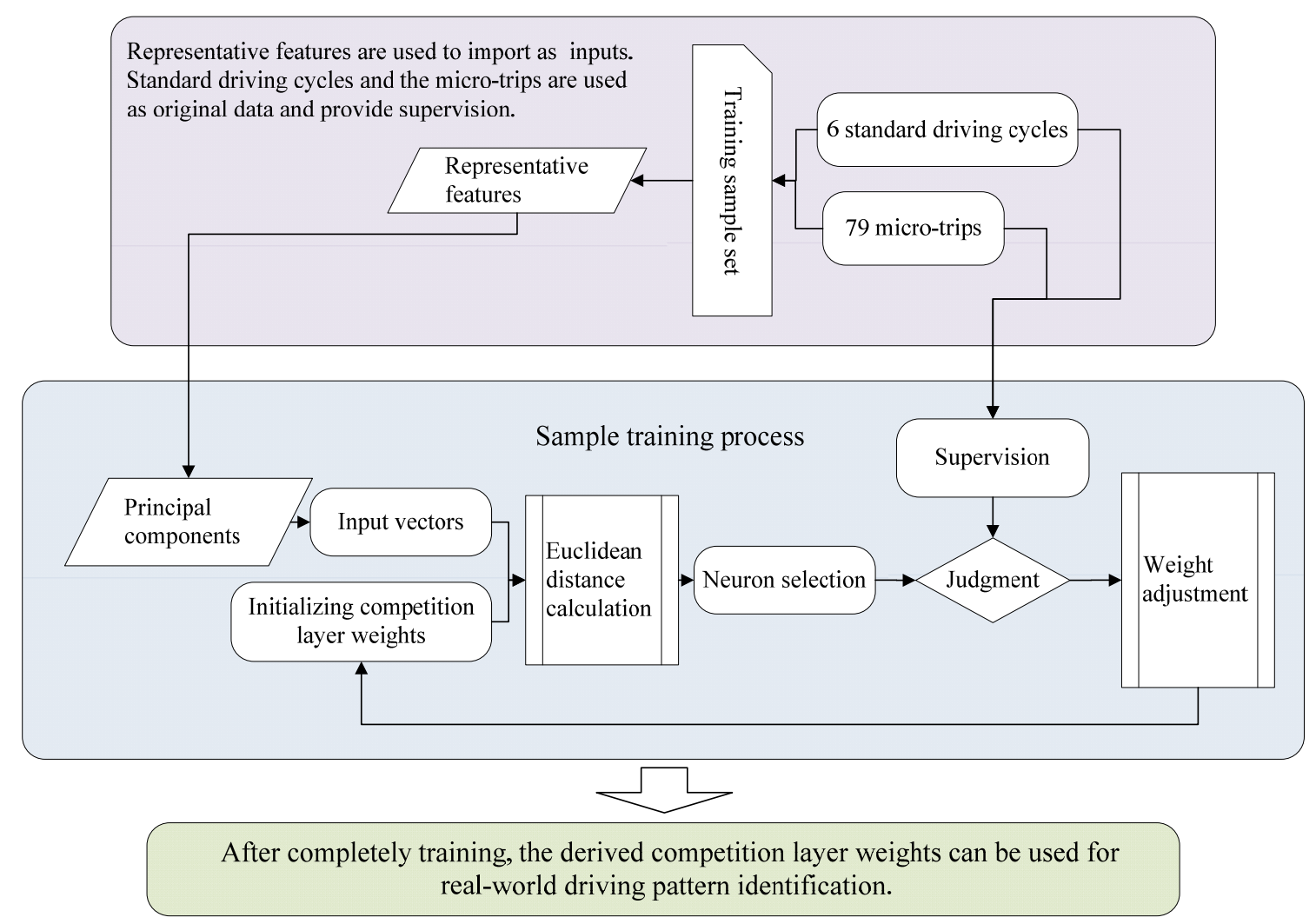

Calculation steps are as follows:

Step 1. Initialize the competition layer weight and the learning efficiency $\eta(\eta>0)$;

Step 2. Enter input vector $X=\left(x_{1}, x_{2}, \cdots, x_{R}\right)^{T}$ into input layer and calculate the Euclidean distance of the competition layer neurons and the input vector according to Equation (14):

$$
d_{i}=\sqrt{\sum_{j=1}^{R}\left(x_{i}-w_{i j}\right)^{2}} \quad i=1,2, \cdots, S^{1}
$$

where: $w_{i j}$-competition layer weight between input layer neuron $j$ and competition layer neuron $i$; 
Step 3. Select two closest competition layer neurons $m$ and $n$. If $d_{m}$ is the minimum distance to input vector, mark the class label of the corresponding linear output layer neuron connected as $C_{m}$. If $d_{n}$ is the second minimum distance to input vector, mark the class label of the corresponding linear output layer neuron connected as $C_{n}$;

Step 4. If neuron $m$ and neuron $n$ can satisfy the following two conditions:

(1) Neuron $m$ and neuron $n$ correspond with different patterns or categories;

(2) The distances (to input vector) $d_{m}$ and $d_{n}$ meet Equation (15):

$$
\min \left\{\frac{d_{m}}{d_{n}}, \frac{d_{n}}{d_{m}}\right\}>\rho
$$

where $\rho$-the window width that the input vector may fall into, usually about $2 / 3$.

Then:

(1) If the category of neuron $m$, supposed as $C_{m}$, is consistent with the input vector category $C_{x}$, the competition weights of neuron $m$ and neuron $n$ will be updated according to Equation (16):

$$
\left\{\begin{array}{l}
w_{m}^{\text {new }}=w_{m}^{\text {old }}+a\left(x-w_{m}^{\text {old }}\right) \\
w_{n}^{\text {new }}=w_{n}^{\text {old }}-a\left(x-w_{n}^{\text {old }}\right)
\end{array}\right.
$$

(2) If the category of neuron $n$, supposed as $C_{n}$, is consistent with the input vector category $C_{x}$, the competition weights of neuron $m$ and neuron $n$ will be updated according to Equation (17):

$$
\left\{\begin{array}{l}
w_{m}^{\text {new }}=w_{m}^{\text {old }}-a\left(x-w_{m}^{\text {old }}\right) \\
w_{n}^{\text {new }}=w_{n}^{\text {old }}+a\left(x-w_{n}^{\text {old }}\right)
\end{array}\right.
$$

Step 5. If neuron $m$ and neuron $n$ can not meet the conditions in Step 4, only the competition weights of the closest neuron $i$ (or $m$ ) will be updated. Suppose the category of neuron $i$ is $C_{x}$ and the correct category of input vector is $C_{i}$. If $C_{i}=C_{x}$ :

$$
w_{i j_{-} \text {new }}=w_{i j_{\_} \text {old }}+\eta\left(x-w_{i j_{-} \text {old }}\right)
$$

or else:

$$
w_{i j_{-} \text {new }}=w_{i j_{\_} \text {old }}-\eta\left(x-w_{i j_{-} \text {old }}\right)
$$

\subsection{Identifier Model Verification}

A composite testing driving cycle of "NYCC+ WVUSUB + HS06_HWY+ UDDS + NewYorkBus+ INDIA_HWY" is established to verify the driving pattern identifier model, as shown in Figure 10. The testing cycle contains three kinds of standard driving cycles which are used for network training and three kinds of new standard driving cycles to increase the credibility of the verification result. The established composite testing driving cycle lasts $5487 \mathrm{~s}$. 
Figure 10. Composite testing driving cycle.

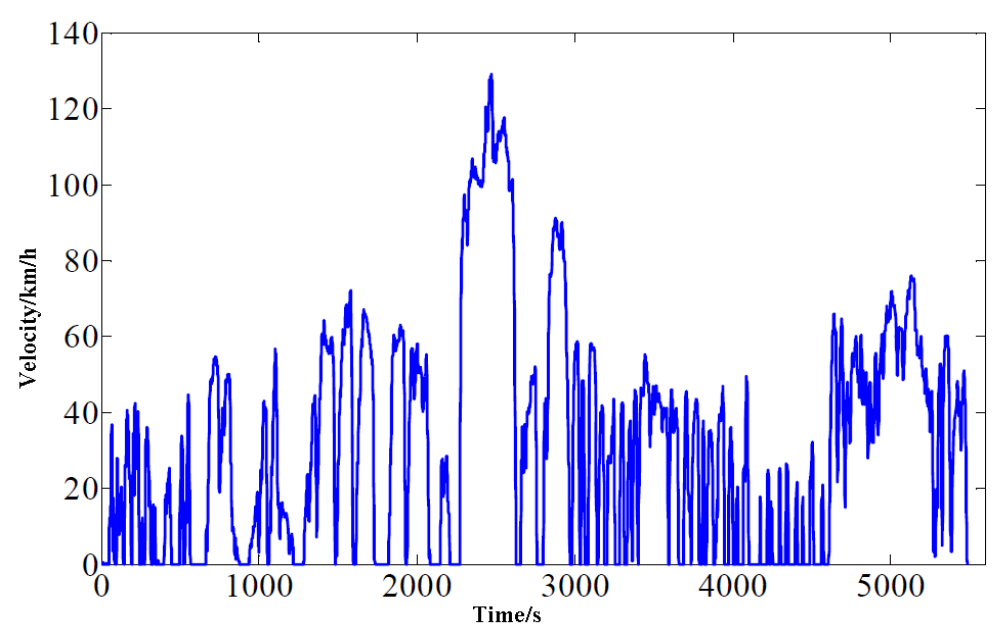

In the first 120 -s phase of the composite testing cycle, the driving cycle category is initialized as " 0 " and the logic threshold parameters are set according to default values. The data collected in the first 120 -s driving phase is used to optimize the logic threshold parameters of the second 120 -s driving phase, and so on. Verification results are illustrated in Figure 11. Contrast Figure 11 with Figure 10, the pattern identification results of different types of driving cycles are relatively more accurate and stable. The identification accuracy of same-type driving cycles is lower.

Figure 11. Driving pattern recognition result.



Main reasons for the identification errors are:

Selected representative features cannot reflect the characteristic differences between similar driving cycles, so feature selection needs to be optimized and the features need to be more representative; competition neurons are insufficient; some of the standard driving cycles, cycles 5-6 for example, are too similar to each other, the recognition of the two cycles is not necessary.

Verification results have proved the validity of the driving cycle identifier model preliminarily, but further validation work is also very necessary. The final evaluation criterion of the performance of the driving pattern identifier is the fuel economy improvement of a HEV, which is the main focus of Section Four. 


\section{Simulation Experiment on the DPR Application}

A co-simulation of the LVQ neural network identifier together with a parallel HEV model [24] is conducted in this section. Input driving cycle is still the composite testing cycle provided by Section Three. The vehicle powertrain and main technical parameters are shown in Figure 12 and listed in Table 4 respectively.

Table 4. Main technical parameters of the parallel HEV powertrain.

\begin{tabular}{lll}
\hline System & Parameter & Value \\
\hline & Curb Weight & $3025 \mathrm{~kg}$ \\
& Front Face Area & $3.042 \mathrm{~m}^{2}$ \\
Vehicle & Rolling Resistance & 0.008 \\
& Air Resistance & 0.4 \\
& Transmission Efficiency & 0.90 \\
& Driving Wheel Rolling Radius & $0.325 \mathrm{~m}$ \\
\hline AMT & Gear Ratio & {$[4.452,2.269,1.517,1,0.854]$} \\
\multirow{2}{*}{ Engine } & Maximum Power Rating & $76 \mathrm{~kW} / 6000$ \\
& Torque Capacity & $131 \mathrm{~N} / 5000$ \\
\hline \multirow{3}{*}{ Motor } & Motor Type & PMSM \\
& Nominal Power Capacity & $10 \mathrm{~kW}$ \\
& Peak Power Capacity & $24 \mathrm{~kW}$ \\
\multirow{2}{*}{ Battery } & Battery Type & Lithium Iron Phosphate \\
& Nominal Voltage & $336 \mathrm{~V}$ \\
& Nominal Capacity & $20 \mathrm{Ah}$ \\
\hline
\end{tabular}

Figure 12. Structure of the parallel HEV powertrain.

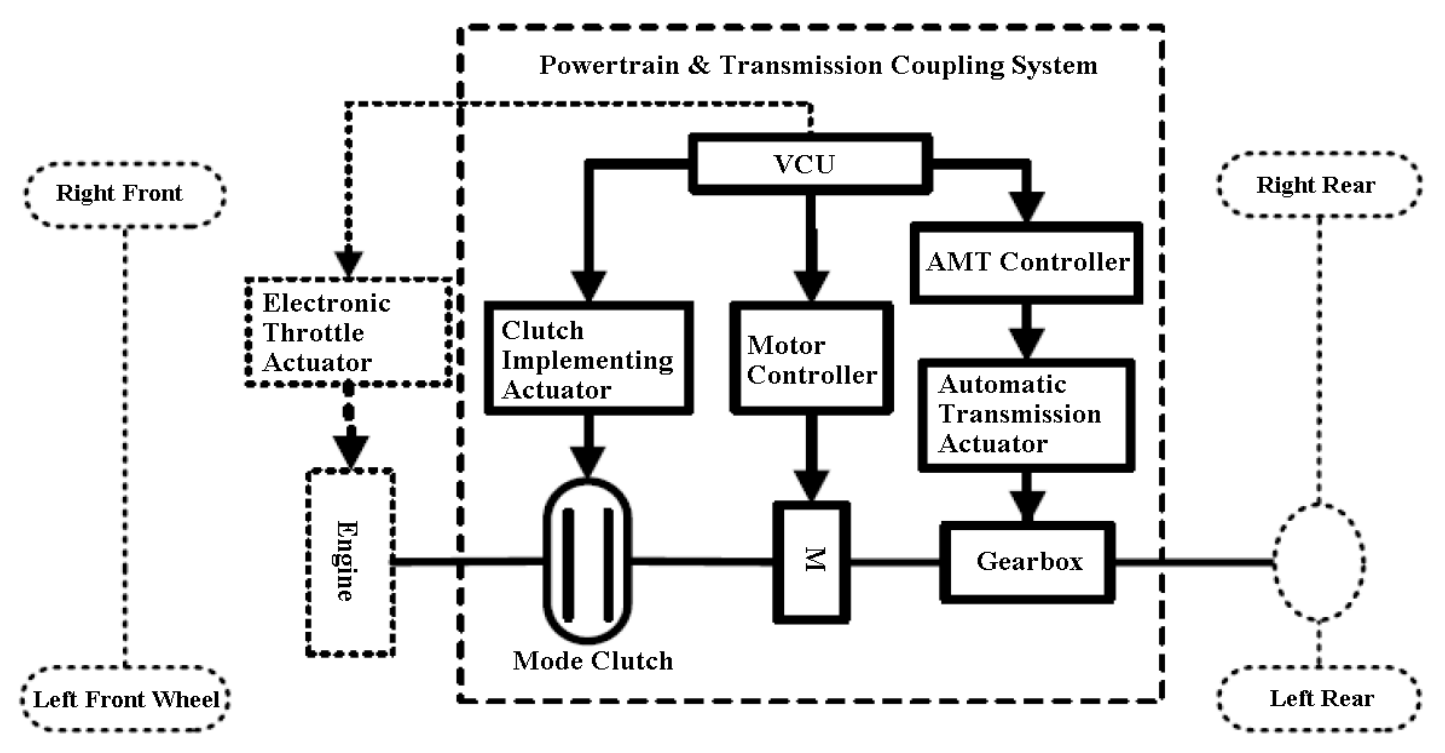

Figure 13 indicates the comparison of velocity data between the testing cycle and the co-simulation result. We can see that the simulation speed follows the testing cycle sensitively, which means that the vehicle has enough power to follow the driving cycle. Moreover, the participation of the driving pattern identifier does not cause any delay of the powertrain control. This provides a good foundation for the comparison study of the control performance with and without DPR optimization. 
Figure 13. Cycle following comparison between the testing cycle and the simulation.

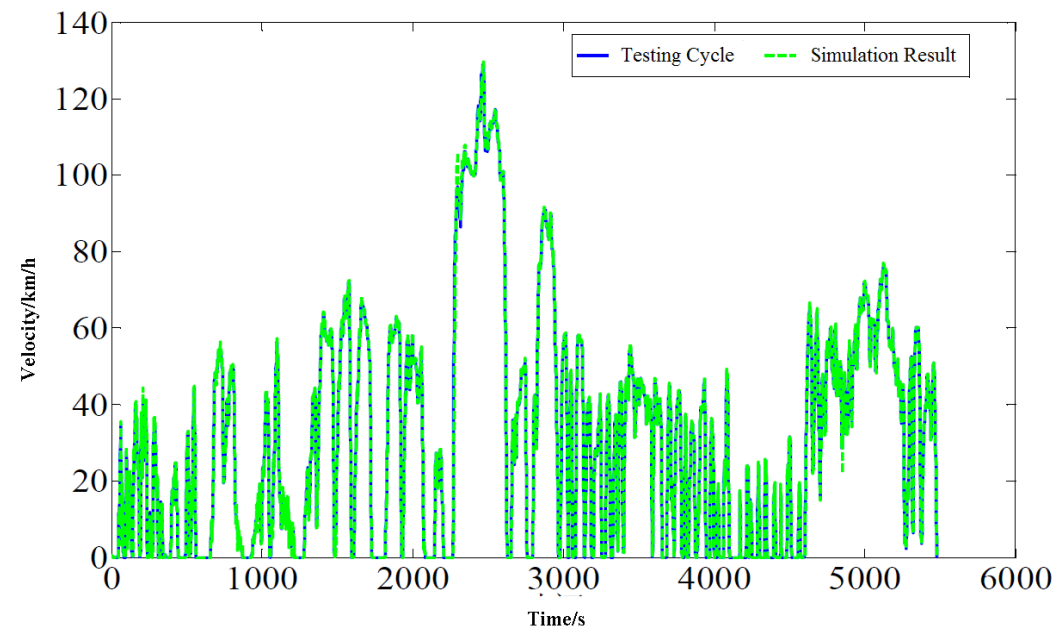

Figure 14 shows the comparison curves of the engine torque, the motor torque results with and without DPR. It can be seen that the engine output torque fluctuates less with DPR optimization than without DPR.

Figure 14. (a) Simulation results of the engine torque; (b) Simulation results of the motor torque.

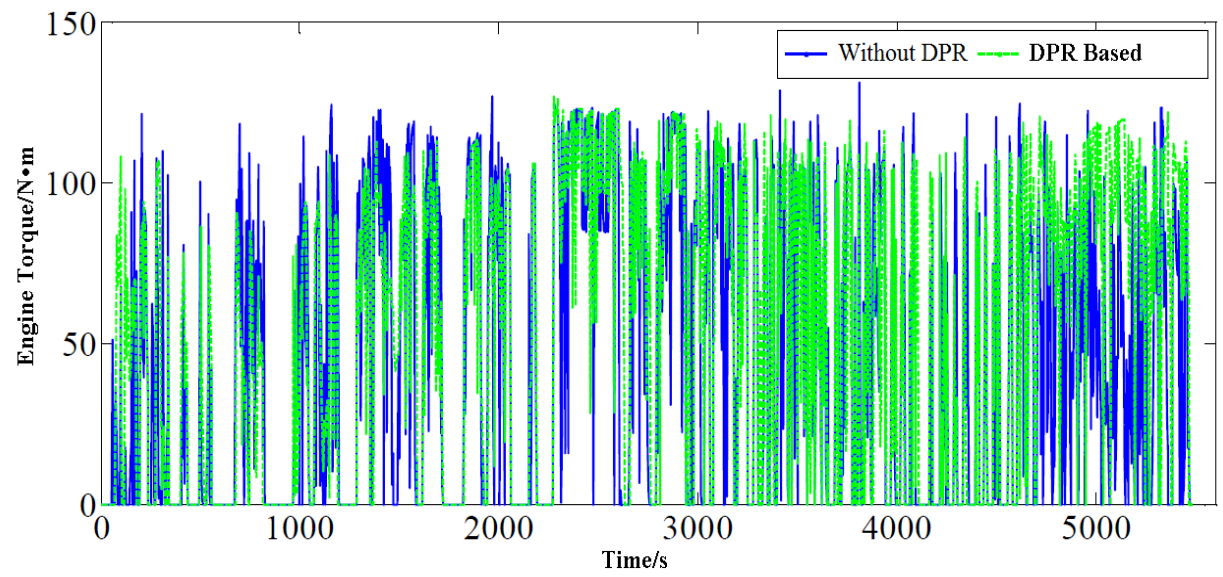

(a)

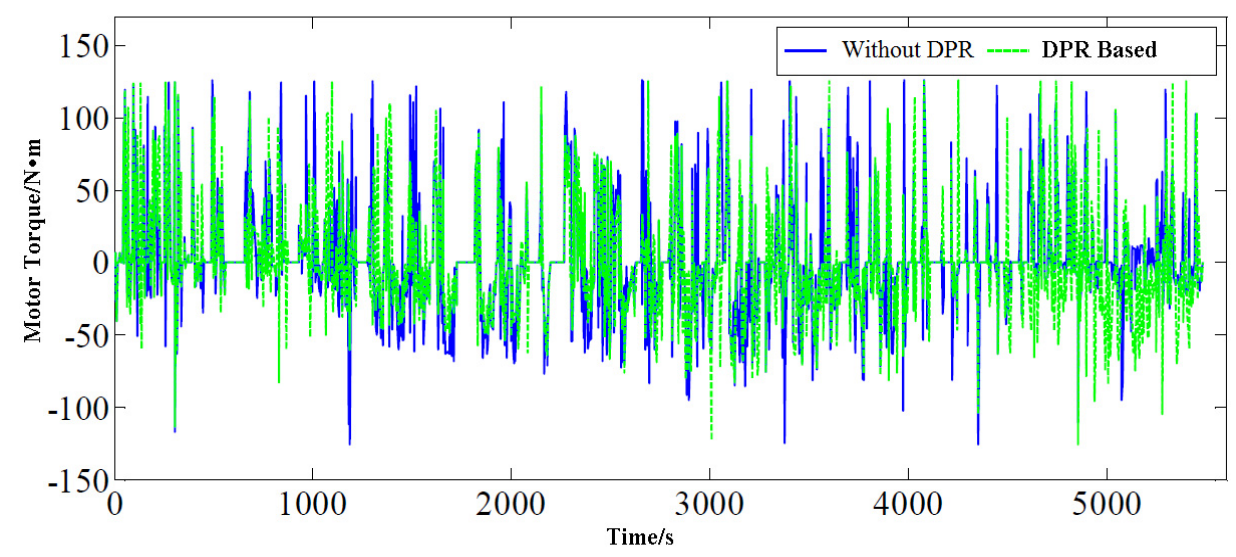

(b) 
The fuel consumption caused by frequent changing of the engine load has decreased due to the optimization of the responsiveness of motor. When the motor is in assisting mode, the torques of the engine and the motor both change little with and without DPR. However, when the motor is in generating mode, the engine outputs more torque to charge the battery with DPR optimization, which can improve the fuel economy globally as a result. Figure 15 shows the distribution map of the engine operating points with and without DPR. Obviously, there are less operating points distributed in the high fuel consumption area after the control algorithm is optimized by DPR.

Figure 15. (a) Engine operating map without DPR; (b) Engine operating map with DPR.

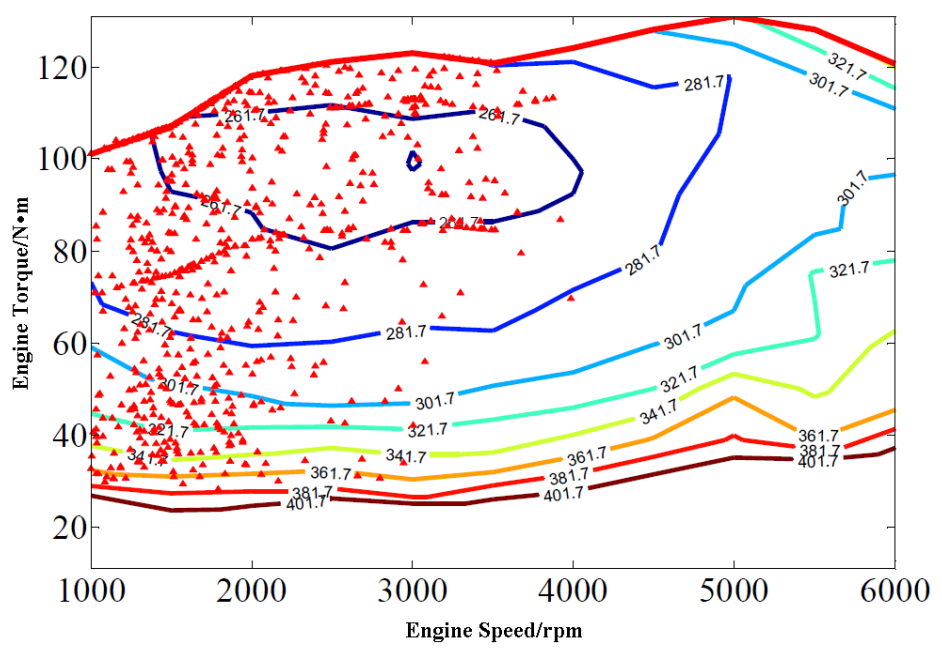

(a)

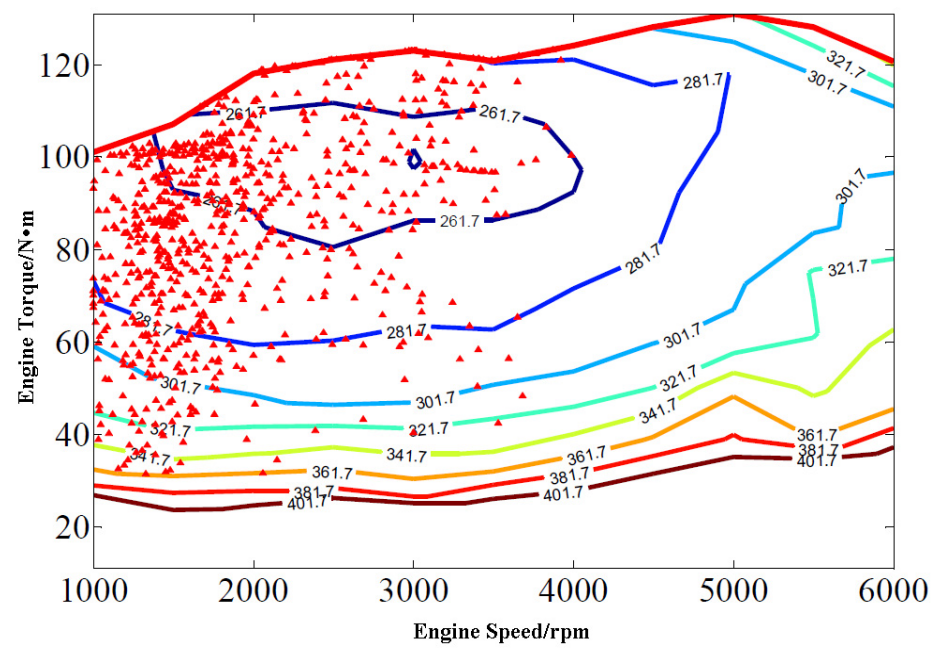

(b)

To illustrate the dependence of the control strategy performance on DPR, an extended application concept of DPR in HEV control is proposed. Firstly control parameters of the standard driving cycles are specially optimized to build a control parameter library, then according to the DPR result, the control parameters, or even control strategies are adjusted in real time based on the optimized control parameter library, and a nearly global optimized control strategy is realized. As a comparison, the specific logic threshold parameters after being optimized based on NYCC are chosen. The fuel consumption results for the above composite testing cycle are listed in Table 5, which shows the 
fuel consumption in case of the DPR optimized is reduced by $8.51 \%$ compared to the case of only the NYCC optimized.

Table 5. Fuel consumption comparison of the DPR optimization.

\begin{tabular}{lll}
\hline & NYCC Optimized & DPR Optimized \\
\hline Fuel Consumption & $10.22 \mathrm{~L} / 100 \mathrm{~km}$ & $9.35 \mathrm{~L} / 100 \mathrm{~km}$ \\
Reduction Ratio & $0.0 \%$ & $8.51 \%$ \\
\hline
\end{tabular}

\section{Conclusions}

An identification method of driving patterns based on LVQ neural network is proposed in this paper to increase the fuel economy of HEVs.

(1) A micro-trip extraction method is used to optimize the training of the LVQ identifier. The amount of training samples is substantially increased and the velocity sampling window length of DPR is reduced within $120 \mathrm{~s}$. As a result, the sampling time is reduced during a recognition process;

(2) Principal Component Analysis (PCA) method is used to optimize the calculation burden of the LVQ identifier. The four principal components extracted from the seven representative features act as the inputs of the LVQ neural network. As a result, the computing space and time of LVQ identifier is reduced and the identification accuracy can still be safeguarded;

(3) Co-simulation of the LVQ driving pattern identifier together with a parallel HEV model is conducted. Simulation results show that the engine works in high-efficiency areas more frequently and the fuel economy can be improved by up to $8.51 \%$.

In the future, hardware-in-the-loop and real-vehicle experiments will be designed and conducted to further verify the recognition effect of the LVQ driving pattern identifier.

\section{Acknowledgements}

This work was supported by the National High Technology Research and Development Program of China (2011AA112304, 2011AA11A228, 2011AA1290) in part, the International Cooperation Research Program of Chinese Ministry of Science and Technology (2011DFB70020) in part, the Program for New Century Excellent Talents in University (NCET-11-0785). The authors would also like to thank the reviewers for their corrections and helpful suggestions.

\section{References}

1. Chan, C.C. The state of the art of electric, hybrid, and fuel cell vehicles. Proc. IEEE 2007, 95, 704-718.

2. Abdelsalam, A.; Cui, S. A fuzzy logic global power management strategy for hybrid electric vehicles based on a permanent magnet electric variable transmission. Energies 2012, 5, 1175-1198. 
3. Gurkaynak, Y.; Khaligh, A.; Emadi, A. State of the Art Power Management Algorithms for Hybrid Electric Vehicles. In Proceedings of the IEEE Vehicle Power and Propulsion Conference, Dearborn, MI, USA, 7-10 September 2009; pp. 388-394.

4. Jeon, S.I.; Jo, S.T.; Park, Y.I.; Lee, J.M. Multimode driving control of a parallel hybrid electric vehicle using driving pattern recognition. J. Dyn. Syst. Meas. Control 2002, 124, 141-149.

5. Lin, C.C.; Jeon, S.; Peng, H.; Lee, J.M. Driving pattern recognition for control of hybrid electric trucks. Veh. Syst. Dyn.: Int. J. Veh. Mech. Mobil. 2004, 42, 41-58.

6. Musardo, C.; Rizzoni, G.; Staccia, B. A-ECMS: An Adaptive Algorithm for Hybrid Electric Vehicle Energy Management. In Proceedings of the 44th IEEE Conference on Decision and Control, Seville, Spain, 12-15 December 2005; pp. 1816-1823.

7. Gong, Q.M.; Li, Y.Y.; Peng, Z.R. Power Management of Plug-in Hybrid Electric Vehicles Using Neural Network based Trip Modeling. In Proceedings of the American Control Conference, St. Louis, MI, USA, 10-12 June 2009; pp. 4601-4606.

8. Zhang, C.; Vahidi, A. Role of terrain preview in energy management of hybrid electric vehicles. IEEE Trans. Veh. Technol. 2010, 59, 1139-1147.

9. Wang, R.; Lukic, S.M. Review of Driving Conditions Prediction and Driving Style Recognition Based Control Algorithms for Hybrid Electric Vehicles. In Proceedings of the 2011 IEEE Vehicle Power and Propulsion Conference (VPPC), Chicago, IL, USA, 6-9 September 2011; Volume 1, pp. 1-7.

10. Won, J.S.; Langari, R. Intelligent energy management agent for a parallel hybrid vehicle-Part II: Torque distribution, charge sustenance strategies, and performance results. IEEE Trans. Veh. Technol. 2005, 54, 935-953.

11. Langari, R.; Won, J.S. Integrated Drive Cycle Analysis for Fuzzy Logic Based Energy Management in Hybrid Vehicles. In Proceedings of the 12th IEEE International Conference on Fuzzy Systems, St. Louis, MI, USA, 25-28 May 2003; pp. 290-295.

12. Won, J.S.; Langari, R. Intelligent energy management agent for a parallel hybrid vehicle_Part I: System architecture and design of the driving situation identification process. IEEE Trans. Veh. Technol. 2005, 54, 925-934.

13. Lei, F.; Liu, W.; Chen, B. Driving pattern recognition for adaptive hybrid vehicle control. SAE Tech. Paper 2012, doi:10.4271/2012-01-0742.

14. Press, W.H.; Teukolsky, S.A. The Art of Scientific Computing, 3rd ed.; Cambridge University Press: New York, NY, USA, 2007; pp. 840-883.

15. Ericsson, E. Independent driving pattern factors and their influence on fuel-use and exhaust emission factors. Transp. Res. Part D Transp. Environ. 2001, 6, 325-345.

16. Tian, Y.; Zhang, X.; Zhang, L. Intelligent Energy Management Based on Driving Cycle Identification Using Fuzzy Neural Network. In Proceedings of the 2nd International Symposium on Computational Intelligence and Design, Changsha, China, 12-14 December 2009; Volume 2, pp. 501-504.

17. Montazeri-Gh, M.; Asadi, M. Intelligent approach for parallel HEV control strategy based on driving cycles. Int. J. Syst. Sci. 2011, 42, 287-302. 
18. Montazeri, M.; Fotouhi, A.; Naderpour, A. Driving segment simulation for determination of the most effective driving features for HEV intelligent control. Veh. Syst. Dyn.: Int. J. Veh. Mech. Mobil. 2012, 50, 229-246.

19. Langari, R.; Won, J.S. A driving situation awareness-based energy management strategy for parallel hybrid vehicles. SAE Trans. 2003, 112, 1938-1947.

20. Xing, J. Study on HEV Genetic-Fuzzy Control Strategy Based On Driving Cycle Recognition. Ph.D. Thesis, Beijing Institute of Technology, Beijing, China, 2010.

21. Mehrotra, K.; Mohan, C.K.; Ranka, S. Elements of Artificial Neural Networks; MIT Press: Cambridge, MA, USA, 1997; pp. 173-180.

22. Chen, H.Y.; Wu, G.Q. Compensation fuzzy neural network power management strategy for hybrid electric vehicle. J. Tongji Univ. (Nat. Sci.) 2009, 37, 525-530.

23. Shi, Z.Z. Neural Networks, 1st ed.; Higher Education Press: Beijing, China, 2009; pp. 152-169.

24. He, H.; Liu, Z.; Zhu, L.; Liu, X. Dynamic coordinated shifting control of automated mechanical transmissions without a clutch in a plug-in hybrid electric vehicle. Energies 2012, 5, 3094-3109.

(C) 2012 by the authors; licensee MDPI, Basel, Switzerland. This article is an open access article distributed under the terms and conditions of the Creative Commons Attribution license (http://creativecommons.org/licenses/by/3.0/). 